\section{Hidradenitis suppurativa in a patient with hyperandro- genism, insulin-resistance and acanthosis nigricans (HAIR-AN syndrome)}

\author{
Yaser Taha Melibary \\ Private Clinic for Dermatology, Allergy \\ and Aesthetic Medicine, Coesfeld, \\ Germany
}

\begin{abstract}
The author reports a rare presentation of hidradenitis supprutiva (HS) in combination with hyperandrogenism, insulin-resistance and acanthosis nigricans (HAIR-AN syndrome) in a female patient. Recently many clues have improved the understanding of HS as a systemic disease rather as a disease only with dermatological manifestation. This report might give another hint emphasizing the endocrinological and hormonal role in HS.
\end{abstract}

\section{Case Report}

An 18-year-old female patient presented to the dermatology clinic with recurring painful and tender boiles on her axillae, groin and neck. She had been suffering from these boiles for two years. Her family history was unremarkable for hidradenitis supprutiva (HS). Previous therapy included local antimycotic treatment (clotrimazole, econazole and ketoconazole), local antiinflammatory treatment with corticosteroids and systemic antibiotic treatment (doxycyclin, clindamycin, rifampicin and azithromycin). The patient is a known case of insulin resistance (laboratory findings: fasting blood sugar of $65 \mathrm{mg} / \mathrm{dL}$ (normal), high fasting insulin level of $20 \mathrm{mU} / \mathrm{L}$ and a positive glucose tolerance test with maximal $195 \mathrm{mg} / \mathrm{dL}$ after $1 \mathrm{~h}$ and $144 \mathrm{mg} / \mathrm{dL}$ after 2h, HbAlc 5.9), consequently Metformin had been administered but discontinued after 12 months because of intolerable side effects (nausea). Cyproterone acetate was also prescribed in order to treat the polycystic ovarian syndrome (PCOS) and hyperandrogenism (DHEA Serum: $4397 \mathrm{ng} / \mathrm{ml}$, free androgen index of 49, normal testosterone and tissue proteins).

On physical examination, the patient had normal vital signs, her body mass index (BMI) was $37.1 \mathrm{~kg} / \mathrm{m}^{2}$ (body height: 185 $\mathrm{cm}$ and body weight: $127 \mathrm{~kg}$ ). She showed seborrhea of the scalp, the face and the upper back. Acanthosis nigricans was found in the axillae, abdominal, submammary, neck and in the groins. A mild form of hypertrichosis presented with growth of beard hair and narrowing the hairline border on the sides of the forehead.

There were multiple inflamed nodes on the neck, the intramammary and inframammary region, both axillae, inguinal folds and one sinus tract draining pus in her left axilla (Figure 1). On the basis of the aforementioned clinical findings, the diagnosis of hidradenitis suppurativa Hurley stage II was made.

Antibiotic treatment with clindamycin $300 \mathrm{mg}$ bd and rifampicin $300 \mathrm{mg}$ bd was administered for 10 weeks. After completion of the prescribed course of antibiotic treatment, the remaining inflammatory lesions of HS on the above-mentioned areas had completely regressed. Then, a wide surgical excision of two inflamed deep-seated nodes, one sinus tract of $2.5 \mathrm{~cm}$ length and one atrophic scar in the left axilla was carried out (Figure 1).

The histopathological examination yielded the following results: an expanded epidermis with deep-seated and expanded hair follicles, surrounded by massive infiltration consisting of neutrophils, plasma cells, lymphocytes, eosinophils and histiocytes. The epithelium of the hair follicles was degraded. In other nearby tissue, we found a moderately cell-rich scar tissue with extensive plasma cell collection surrounded by vascularity.

Subsequently, she was referred to the endocrinology clinic where congenital adrenal hyperplasia, Cushing syndrome and hypercortisolism were excluded.

\section{Discussion}

HAIR-AN-syndrome is an acronym for a multi-systemic condition that affects women and consists of hyperandrogenism (HA), insulin-resistance (IR) and acanthosis nigricans (AN). It is understood as a subphenotype of PCOS, however it differs from the PCOS by the presence of insulin resistance. It is associated with psychological distress, depression and low selfesteem. ${ }^{1,2}$

The hyperandrogenism manifests with male body habitus, acne, menstrual irregularities, hirsutism and seborrhea. AN develops due to long term sensitization to elevated insulin, which binds to IGF-1 receptors on the keratinocytes, thereby stimulating the proliferation of the epidermal keratinocytes and dermal fibroblasts. ${ }^{3}$ Hyperinsulinemia and Insulin resistance
Correspondence: Yaser T. Melibary, Private Clinic for Dermatology, Allergy and Aesthetic Medicine, Rosenstr. 1 /Markt 1, Dermatologische GP Dr. Pappai und Prof. Schiller, Coesfeld 48653, Germany.

E-mail: ymelibary@gmail.com

Key words: Hidradenitis suppurativa; acne inversa; HAIR-AN syndrome; polycystic ovarian syndrome (PCOS).

Acknowledgments: the author would like to thank Dr. M. Knut; Clinic of Endocrinology, Charité-University Hospital Berlin, Germany, Dr. W. Alotaibi, Maternity and Children Hospital, Makkah, Saudi Arabia and Dr. S. Schneider-Burrus; Department of Dermatology, Venereology and Allergology, Charité-University Hospital Berlin and Havelklinik Berlin, Germany.

Conflicts of interest: the author declares no potential conflict of interest.

Received for publication: 19 December 2017. Accepted for publication: 4 June 2018 .

This work is licensed under a Creative Commons Attribution-NonCommercial 4.0 International License (CC BY-NC 4.0).

(C) Copyright Y.T. Melibary, 2018

Licensee PAGEPress, Italy

Dermatology Reports 2018; 10:7546

doi:10.4081/dr.2018.7546

have often an impact on each other and they may induce one another. ${ }^{4}$ Monfrecola et al. ${ }^{5}$ suggested that the mTOR dysregulation (conserved serine/threonine kinase mammailian target of rapamycin) may play a role in the inflammatory background of HS and might be a marker of its severity on the insulin- resistance HS patients. On the other hand, the role of hyperandrogenism in terms of high free androgen index and high DHEA-serum and the sexual hormones and the definitive improvement effect under antiandrogen therapy are still in different studies conflictual. ${ }^{6-9}$ Under these complicated loop effects of obesity, androgen production and insulin resistance manifest the symptoms of HS further. Nevertheless, Elmer et al. ${ }^{3}$ mentioned the possibility for underlying endocrine conditions that may associate HAIR-AN-syndrome.

Hidradenitis suppurativa (HS) is a chronically recurring inflammatory disease that affects mostly the axillae, groin, perineum, anogenital and inframammary regions. Women seem to be affected more often by HS than men with the course of disease being more severe in men than women. ${ }^{6}$ Genetic predisposition appears to be a risk factor for HS with a positive fam- 
ily history for the disease in about a third of all patients. ${ }^{10}$ Mutations of the $\gamma$-secretase gene and its a cofactor subunit: nicastrin (NCSTN) and High Copy Numbers of $\beta$ Defensin Cluster on 8p23.1 have been allocated in patients with severe forms familyassociated HS. ${ }^{10-13}$ Several modifiable behavioral risk factors are known for HS, in particular cigarette smoking and obesity. Since a number of comorbid conditions like the metabolic syndrome, chronic inflammatory bowel disease and psychosomatic disorders have been strongly associated with HS, the disease is today understood as a systemic disorder. ${ }^{10,14-17}$ Nevertheless its etiology is still not completely understood.

Earth et al. ${ }^{18}$ studied 13 cases with acanthosis nigricans (AN) who have presented with features of cutaneous virilism, he examined 5 patients in that group suffered from HS without any further written clarifications in regard of any relationship between their symptoms, and it was shown in another study on 70 females by Jemec et $a l .{ }^{19}$ that HS is not associated with other signs of androgenization. Therefore, It is unclear, if our patient and those patients in the study by Earth et al. developed HS because of the elevated risk factors for HS (being overweight, insulin resistance and hyperandrogenism), but since in Earth's group of 13 patients 5 were suffering from HS, HAIR-AN seems to be an independent risk factor for HS. Stone et al. ${ }^{20}$ reported in 1976 another association between HS and $\mathrm{AN}$ in male patients was reported in a case. Mostly, this association was explained by the common cause for both disorders being obesity. Whether the etiology in our case is due to obesity or insulin resistance or to

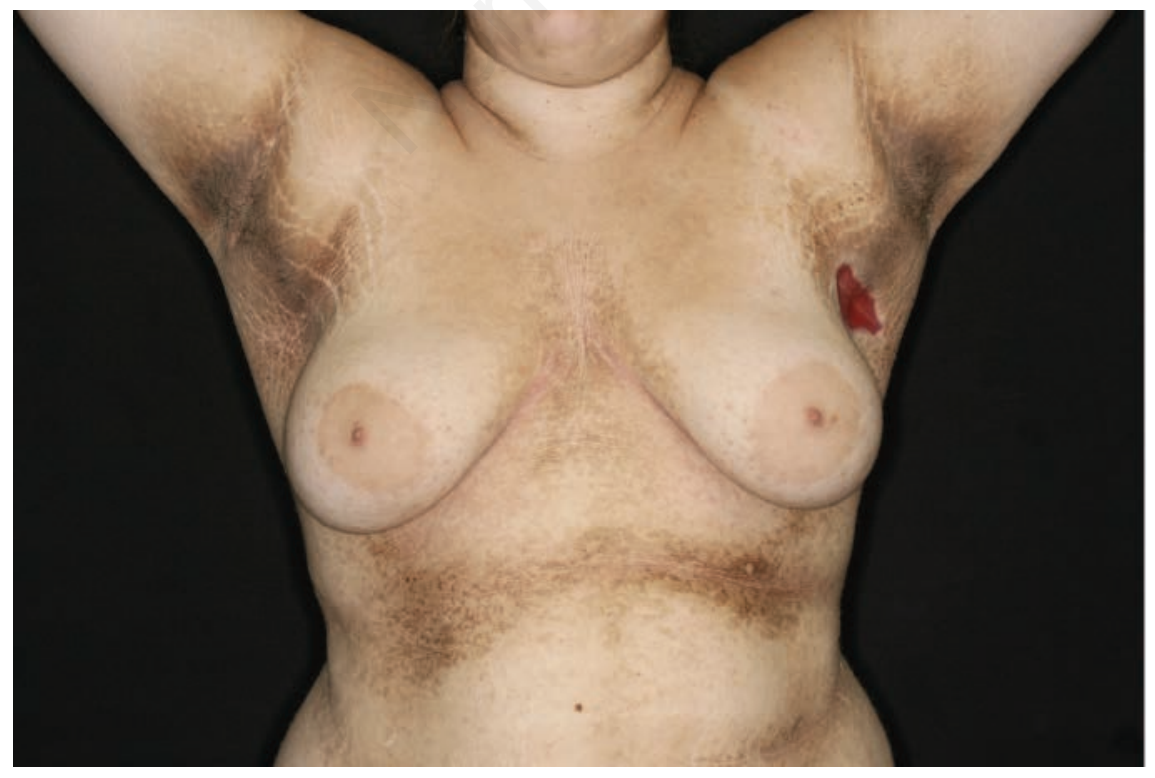

Figure 1. Acanthosis nigrans on different body folds (photo was taken post operative). unclear pathological association between to AN or secondary to all of them, is still unclear. Many characteristics such as its postpubertal onset, female predominance, pre-menstrual flare ups and perigestational improvement, indicate that hormonal disturbance with or without the endocrinological abnormalities play an unclear provoking role in the emergence of HS. ${ }^{8}$ Thus it has not been mentioned in the literature any previous presentation with these manifestations of HAIR-AN and HS together rather in male or female patients, this case reports the first association between HAIR-AN syndrome and HS in a female patient. However, it stays unclear which etiological background provokes the other manifestations. Nevertheless, this case strengthens the indications of possible associations with HS to hormonal dysregulation.

\section{Conclusions}

Hidradenitis suppurativa showed itself with multifactorial presentations and often with different systemic relations. Definitely, more studies are needed to evaluate the endocrinological and hormonal role in the emergence of HS. This case might suggest a new association that requires further investigation. It is still unclear if an etiological association presents between HS with HAIR-AN-syndrome as a whole or in part. Nevertheless, it is important for physicians treating patients with HAIR-AN syndrome to pay attention to early symptoms of HS to improve diagnostic accuracy and disease management.

\section{References}

1. Omar HA, Logsdon S, Richards J. Clinical profiles, occurrence, and management of adolescent patients with HAIR-AN syndrome. Sci World J 2004;4:507-11.

2. Eiser C, Morse R. Quality-of-life measures in chronic diseases of childhood. Health Technol Assess (Winchester, England) 2001;5:1.

3. Elmer KB, George RM. HAIR-AN syndrome: a multisystem challenge. Am Fam Phys 2001; 63:2385-90.

4. Shanik MH, Xu Y, Škrha J. Insulin resistance and hyperinsulinemia. Diabet Care 2008;31:262-8.

5. Monfrecola B, Balato A, Caiazzo G, et al. Mammalian target of rapamycin, insulin resistance and hidradenitis suppurativa: a possible metabolic loop. J Eur Acad Dermatol Venereol 2016;30:1631-3.

6. Micheletti RG. Hidradenitis suppurativa: current views on epidemiology, pathogenesis, and pathophysiology. Semin Cutan Med Surg 2014;33:48-50.

7. Harrison BJ, Kumar S, Read GF. Hidradenitis suppurativa: evidence for an endocrine abnormality. Br J Surg 1985;72:1002-4.

8. Karagiannidis I, Nikolakis G, Sabat R, Zouboulis CC. Hidradenitis suppurativa/Acne inversa: an endocrine skin disorder?. Rev Endocr Metab Disord 2016;17:335-41.

9. Mortimer PS, Dawber RP, Gales MA, Moore RA. Mediation of hidradenitis suppurativa by androgens. Br Med J (Clin Res Ed) 1986;292:245-8.

10. Zouboulis CC, Desai N, Emtestam L, et al. European S1 guideline for the treatment of hidradenitis suppurativa/acne inversa. J Eur Acad Dermatol Venereol 2015;29:619-44.

11. Giamarellos-Bourboulis EJ, Platzer M, Karagiannidis I, et al. High copy numbers of $\beta$-defensin cluster on 8p23. 1, Confer Genetic Susceptibility, and Modulate the Physical Course of Hidradenitis Suppurativa/Acne Inversa. J Invest Dermatol 2016;136:1592-8.

12. Pink AE, Simpson MA, Desai N, et al. Mutations in the [gamma]-secretase genes NCSTN, PSENEN, and PSEN1 underlie rare forms of hidradenitis suppurativa (acne inversa). J Invest Dermatol 2012;132:2459.

13. Xiao X, He Y, Li C, et al. Nicastrin mutations in familial acne inversa impact keratinocyte proliferation and differentiation through the Notch and phosphoinositide 3-kinase/AKT sig- 
nalling pathways. $\mathrm{Br} \mathrm{J}$ Dermatol 2016;174:522-32.

14. Pink AE, Simpson MA, Desai N, et al. $\gamma$-Secretase mutations in hidradenitis suppurativa: new insights into disease pathogenesis. J Invest Dermatol 2013;133:601-7.

15. Van der Zee HH, Laman JD, Boer J, Prens EP. Hidradenitis suppurativa: viewpoint on clinical phenotyping, pathogenesis and novel treatments.
Exper Dermatol 2012;21:735-9.

16. Sabat R, Chanwangpong A, SchneiderBurrus S, et al. Increased prevalence of metabolic syndrome in patients with acne inversa. PLoS One 2012; 7:e31810.

17. Shalom G, Freud T, Harman-Boehm I, et al. Hidradenitis suppurativa and metabolic syndrome: a comparative cross-sectional study of 3207 patients. Br J Dermatol 2015;173:464-70.

18. Earth J, Ng LL, Wojnarowska F,
Dawber RPR. Acanthosis nigricans, insulin resistance and cutaneous virilism. Br J Dermatol 1988;118:613-9.

19. Jemec GBE. The symptomatology of hidradenitis suppurativa in women. Br J Dermatol 1988;119:345-50.

20. Stone OJ. Hidradenitis suppurativa following acanthosis nigricans: report of two cases. Archiv Dermatol 1976;112: 1142. 